\title{
The Crystal Orientation Taking Account of Gravity Force under High Magnetic Field
}

\author{
Cunyou WU, Shuqin LI, Kensuke SASSA, Yoshio SAKKA, ${ }^{1)}$ Tohru S. SUZUKI") and Shigeo ASAI \\ Department of Materials, Physics and Energy Engineering, Graduate School of Engineering, Nagoya University, Furo-cho, \\ Chikusa Nagoya 464-8603 Japan. $\quad$ 1) National Institute for Materials Science, 1-2-1, Sengen, Tsukuba, Ibaraki $30-0047$ \\ Japan.
}

(Received on December 2, 2004; accepted on February 15, 2005)

\begin{abstract}
High magnetic field is well known to have the function of crystal orientation, which is largely influenced by crystal size and shape to some extent. For crystals with large rod shape, the gravity force functions and affects the crystal orientation. In our experiment, different results were got when a high magnetic field was applied perpendicular and parallel to the gravity force. In this paper, the effect of the gravity force for crystal orientation under the high magnetic field is summarized and compared with the experiment result.
\end{abstract}

KEY WORDS: electromagnetic processing of materials; crystal orientation; high magnetic field; gravity force; slip casting.

\section{Introduction}

It is well known that crystals with magnetic anisotropy can be oriented by high magnetic field. The principle of crystal orientation using the high magnetic field is that a torque due to magnetic anisotropy rotates a crystal so as to take a stable crystal orientation and decrease magnetization energy. ${ }^{1,2)}$ For crystals with different anisotropic magnetic susceptibility, the orientation directions are different.

The application of the high magnetic field has now been recognized as one of the useful technologies in materials processing to get texture structures. ${ }^{3-6)}$ Among all the researches reported hitherto, the effect of the high magnetic field on crystal orientation has been confirmed and well utilized. However, few results show highly crystal orientated structure. On the other hand, regarding crystal orientation under a high magnetic field, the effect of the gravity force also plays an important role when it is related with a crystal shape. However, the effect of the gravity force has for long time been neglected.

In our research, the experimental results were determined by the imposed direction of the high magnetic field, which indicates the necessary of considering the gravity force during crystal orientation. Therefore, we summarize the expected crystal orientation in each direction of a sample when both of the magnetic field and the gravity force are taken into account, and also that without considering the gravity force.

\section{Experiment}

Slip casting of titanium whisker under a high magnetic field was conducted. ${ }^{7)}$ The titanium whisker has a rod-like morphology with a mean length of $1.68 \mu \mathrm{m}$ and diameter of
$0.13 \mu \mathrm{m}$. The structure is rutile-type single crystal and the long-axis is confirmed to be $c$-axis based on its electron diffraction pattern. The titanium whiskers were dispersed in distilled water by adding the appropriate amount (2.46 mass $\%$ addition to the titanium whisker) of polyelectrolyte with the aid of ultrasonic irradiation. The solid titanium content was fixed as $30 \mathrm{vol} \%$. A high magnetic field of $10 \mathrm{~T}$ was applied parallel and perpendicular to the direction of the gravity force. In comparison, normal colloidal filtration without magnetic field was also conducted. The green compacts were heated at $1073 \mathrm{~K}$ for $2 \mathrm{~h}$ for the convenience of treatment. The heated body was cut in horizontal and vertical cross sections to the imposed magnetic field.

To quantitatively clarify the difference, X-ray diffraction data were analyzed by the orientation index using the three main peaks of (110), (101) and (002). The orientation index on each cut plane was calculated based on the X-ray diffraction pattern. The orientation index of the $\left(h_{i} k_{i} l_{i}\right)$ plane $N_{h_{i} k_{i} l_{i}}$ defined by Eq. $(1)^{8)}$ is evaluated from the X-ray diffraction patterns.

$$
N_{h_{i} k_{i} l_{i}}=\frac{F_{h_{i} k_{i} l_{i}}}{F_{h_{i} k_{i} l_{i}}^{0}}
$$

where $F_{h_{i} k_{i} l_{i}}$ is an intensity fraction of a $\left(h_{i} k_{i} l_{i}\right)$ plane and defined by Eq. (2), and $F_{h_{i} k_{i} l_{i}}^{0}$ is obtained from the standard data of JCPDS card.

$$
F_{h_{i} k_{i} l_{i}}=\frac{I_{h_{i} k_{i} l_{i}}}{I_{h_{1} k_{1} l_{1}}+I_{h_{2} k_{2} l_{2}}+I_{h_{3} k_{3} l_{3}}+\cdots+I_{h_{n} k_{n} l_{n}}}
$$

where $I_{h_{i} k_{i} l_{i}}$ is intensity for the diffraction line of $\left(h_{i} k_{i} l_{i}\right)$. 


\section{Results and Discussion}

\subsection{Theoretical Consideration}

The definition of each surface of a sample is schematically shown in Fig. 1. When a magnetic field is perpendicular to the gravity force (refer to Pe), the crystal orientation in side 1 and side 2 planes are different. While in parallel magnetic field (refer to $\mathrm{Pa}$ ), the side 1 and side 2 in the samples are equivalent and both showed similar X-ray diffraction patterns; therefore in the samples treated by $\mathrm{Pa}$ magnetic field, only the data of side instead of side 1 and side 2 will be shown.

Theoretical study ${ }^{9)}$ on classification of crystal size for orientation under high magnetic field shows that, for fine particles within the size range of

$$
r_{\min }\left(=\sqrt[3]{\frac{3 k T \mu_{0}}{2 \pi \Delta \chi B^{2}}}\right)<r<r_{\mathrm{cri}}\left(=\sqrt[7]{\frac{243 k T \eta^{2}}{8 \pi e g^{2} \rho_{\mathrm{p}}\left(\rho_{\mathrm{p}}-\rho_{1}\right)^{2}}}\right)
$$

the gravity motion and Brownian motion will not attribute to the crystal orientation under high magnetic field. While, for large particles within the size range of

$$
r_{\text {cri }}<r<r_{\max }\left(=\sqrt{\frac{3 \Delta \chi B^{2} L}{4 \mu_{0} g\left(\rho_{\mathrm{p}}-\rho_{1}\right)}}\right)
$$

the gravity force should be taken into account. Here, $g$ is the acceleration of gravity, $r$ the radius of the particle, $\eta$ the viscosity coefficient of a medium, $\rho_{1}$ the density of the medium, $\rho_{\mathrm{p}}$ the density of the particle, $\Delta \chi$ the difference between magnetic susceptibilities in crystal axes, $B$ the imposed magnetic flux density, $k$ Boltzmann constant $\left(1.38 \times 10^{-23} \mathrm{~J} / \mathrm{K}\right), T$ the temperature, $\mu_{0}$ the permeability in vacuum $\left(4 \pi \times 10^{-7}[\mathrm{H} / \mathrm{m}]\right), L$ falling length of the crystal. This theoretical classification indicates the necessary of considering gravity force during crystal orientation under high magnetic field.

The effect of high magnetic field is closely related with the magnetic susceptibilities of crystals, while the effect of the gravity force largely depends on crystal size and shape. According to the theoretical classification of crystal size, for fine crystals, the gravity force will not attribute to the rotation of the crystals. And only the effect of the high magnetic field needs to be considered. As we known, for crystals with $\chi_{c}>\chi_{a, b}\left(\chi_{c}\right.$ and $\chi_{a, b}$ are $c$-axis and $a$ - or $b$-axis of a magnetic susceptibility, respectively), $c$-axis is the preferred direction in parallel to the magnetic field direction, while $a, b$-axis perpendicular to the magnetic field direction. Therefore, according to Fig. 1, only $c$ plane will appear on side 2 plane of a sample in Pe magnetic field, while $a, b$ planes appear on top and side 1 planes. And in the case of Pa magnetic field, only $c$ plane should appear on the top plane while $a, b$ plane appear on the side 1 and side 2 planes. For the crystals with $\chi_{c}<\chi_{a, b}, a$ - (or $b$-) axis is the preferred direction in parallel to the magnetic field direction, while $c$-axis perpendicular to the magnetic field direction. Therefore, according to Fig. 1, $a, b$ planes will appear in the side 2 plane while all of $a, b$ and $c$ planes appear in the top and side 1 planes in Pe magnetic field. Thus, it is

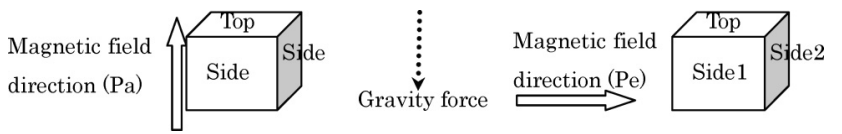

Fig. 1. The definition of surfaces.

Table 1. The expected crystal orientation of the materials in each case when the effect of the gravity force is not considered.

\begin{tabular}{|c|c|c|c|c|c|c|}
\hline \multirow{2}{*}{$\begin{array}{c}\text { Magnetic field } \\
\text { direction }\end{array}$} & \multicolumn{3}{|c|}{$\chi_{c}>\chi_{a, b}$} & \multicolumn{3}{c|}{$\chi_{c}<\chi_{a, b}$} \\
\cline { 2 - 7 } & Top & Side1 & Side2 & Top & Side1 & Side2 \\
\hline $\mathrm{Pe}(\perp)$ & \multicolumn{2}{|c|}{$\mathrm{a}, \mathrm{b}$} & $\mathrm{c}$ & \multicolumn{2}{|c|}{$\mathrm{a}, \mathrm{b}, \mathrm{c}$} & $\mathrm{a}, \mathrm{b}$ \\
\hline $\mathrm{Pa}(/ /)$ & $\mathrm{c}$ & \multicolumn{2}{|c|}{$\mathrm{a}, \mathrm{b}$} & $\mathrm{a}, \mathrm{b}$ & \multicolumn{2}{c|}{$\mathrm{a}, \mathrm{b}, \mathrm{c}$} \\
\hline
\end{tabular}

Table 2. The expected crystal orientation of the materials in each case when the effect of the gravity force is con-

\begin{tabular}{|c|c|c|c|c|c|c|c|}
\hline \multirow{2}{*}{$\begin{array}{l}\text { Crystal } \\
\text { shape }\end{array}$} & \multirow{2}{*}{$\begin{array}{c}\text { Magnetic field } \\
\text { direction }\end{array}$} & \multicolumn{3}{|c|}{$\chi_{c}>\chi_{a, b}$} & \multicolumn{3}{|c|}{$\chi_{c}<\chi_{a, b}$} \\
\hline & & Top & Side1 & Side2 & Top & Side1 & Side2 \\
\hline \multirow{2}{*}{ Spherical } & $\operatorname{Pe}(\perp)$ & \multicolumn{2}{|c|}{$a, b$} & $\mathrm{c}$ & \multicolumn{2}{|c|}{$a, b, c$} & $a, b$ \\
\hline & $\mathrm{Pa}(/ /)$ & $\mathrm{c}$ & \multicolumn{2}{|c|}{$a, b$} & $a, b$ & \multicolumn{2}{|c|}{$a, b, c$} \\
\hline \multirow{2}{*}{ Rod } & $\mathrm{Pe}(\perp)$ & \multicolumn{2}{|c|}{$a, b$} & $\mathrm{c}$ & $a, b$ & $\mathrm{c}$ & $a, b$ \\
\hline & $\mathrm{Pa}(/ /)$ & \multicolumn{3}{|c|}{$a, b, c$} & $a, b$ & \multicolumn{2}{|c|}{$a, b, c$} \\
\hline \multirow{2}{*}{ Plane } & $\mathrm{Pe}(\perp)$ & $\mathrm{a}, \mathrm{b}, \mathrm{c}$ & $a, b$ & $a, b, c$ & $\mathrm{c}$ & \multicolumn{2}{|c|}{$a, b$} \\
\hline & $\mathrm{Pa}(/ /)$ & $\mathrm{c}$ & \multicolumn{2}{|c|}{$a, b$} & \multicolumn{3}{|c|}{$\mathrm{a}, \mathrm{bc}$} \\
\hline
\end{tabular}
sidered.

impossible to uniaxially orient the crystals by simply imposing a high magnetic field. It is suggested that uniaxially oriented structure can be obtained by rotating the magnetic field. However, this method is very difficult to conduct in practical experiment. Thus, a novel process where a specimen is rotated during a slip casting under a high magnetic field has been proposed. ${ }^{10)}$ In the case of Pa magnetic field, $a, b$ planes should appear on the top plane while all of $a, b$ and $c$ planes appear on the side 1 and side 2 planes. Thus the expected crystal orientation in each case can be summarized as Table 1.

The effect of the gravity force for crystal orientation has already been analyzed ${ }^{9}$ ) and it is related with a crystal shape. For a crystal in spherical shape, the gravity force will not attribute to its rotation. Therefore, the expected crystal orientation is same as that shown in Table 1. However, for crystals in rod or plane shapes, the expected crystal orientation will greatly be affected by the gravity force. Table 2 shows the expected crystal orientation of the materials in each case when the effect of the gravity force is considered.

A rod crystal prefers to lie down with its long axis (caxis) perpendicular to the gravity force. On the other hand, crystals with $\chi_{c}>\chi_{a, b}$ will align with its long axis perpendicular the gravity force in Pe magnetic field, while it parallel to the gravity force in Pa magnetic field. Therefore, the gravity force reinforces the orientation effect of Pe magnetic field, however, it counteracts the orientation effect of $\mathrm{Pa}$ magnetic field. That is, the expected crystal orientation in 
Pe magnetic field is same as that without considering the gravity force, while crystal orientation can not be obtained by $\mathrm{Pa}$ magnetic field and all of $a, b$ and $c$ planes appear on each plane of a sample. For the case of $\chi_{c}<\chi_{a, b}$, the crystal orientation becomes much more complicated. The gravity force reinforces the orientation effect of $\mathrm{Pa}$ magnetic field, however, it crossovers with the orientation effect of Pe magnetic field. Therefore, when Pe magnetic field is imposed, $a$, $b$ planes will appear in top and side 2 surfaces, while only $c$ plane appear in side 1 surface. That is, it is possible to get uniaxially oriented crystal structure by imposition of a Pe magnetic field. On the other hand, $a, b$ planes will appear in top surface and $a, b$ and $c$ planes in the side surfaces by imposition of $\mathrm{Pa}$ magnetic field.

In addition, since a plane crystal prefers to lie down with its long axis ( $a, b$-axis) perpendicular to the gravity force, the orientation effect of the gravity force for crystals in plane shape is opposite with that for rod shape, as shown in Table 2. For instance, $c$-axis oriented crystal structure can only be obtained by imposing Pa magnetic field but not Pe magnetic field.

\subsection{Experimental Confirmation}

In order to verify the theoretical analysis given in Table 2 , we have carried out the slip casting of rod shape titanium whisker, and their magnetic susceptibilities have been confirmed to be $\chi_{c}>\chi_{a, b}{ }^{11,12)} \mathrm{X}$-ray diffraction patterns of titanium whisker treated under the high magnetic field of $10 \mathrm{~T}$ are shown in Fig. 2. The orientation indices of the peaks of (110), (101) and (002) and schematic illustration of alignment of titanium whisker are shown in Fig. 3. The sample without magnetic field and one with $\mathrm{Pa}$ magnetic field show almost same orientation indices, while the sample with Pe magnetic field shows high crystal orientation in the side 2 plane.

As shown in Fig. 2(a) obtained without magnetic field, the intensity of (110) peak in the side plane is smaller than that of the top plane, while the intensity of the (002) peak in the side plane corresponding to $c$-axis is higher than the top plane one. That is, the $c$-axes of crystals prefer to lie down and perpendicular to the gravity force. This means that $c$-axis in the long axis of the titanium whisker is randomly distributed on the plane perpendicular to the gravity force. Such a weak alignment of the whisker is usually observed because the gravity force is predominant in the dis-
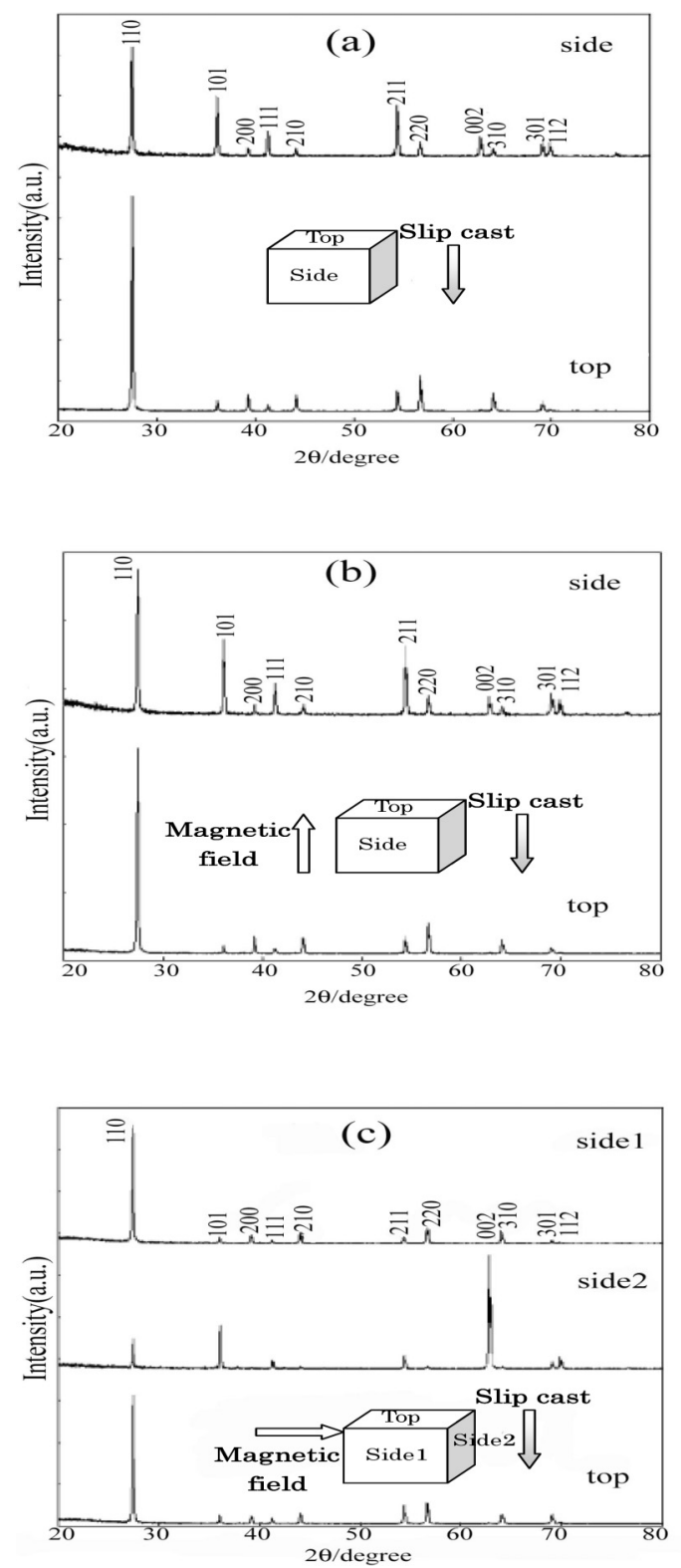

Fig. 2. X-ray diffraction patterns of the samples compacted by colloidal filtration without a magnetic field and in $10 \mathrm{~T}$, followed by heating at $1073 \mathrm{~K}$ for $2 \mathrm{~h}$. The samples are consolidated (a) without a magnetic field, (b) in the magnetic field parallel to the fluid flow, and (c) in the magnetic field perpendicular to the fluid flow (direction of gravity).
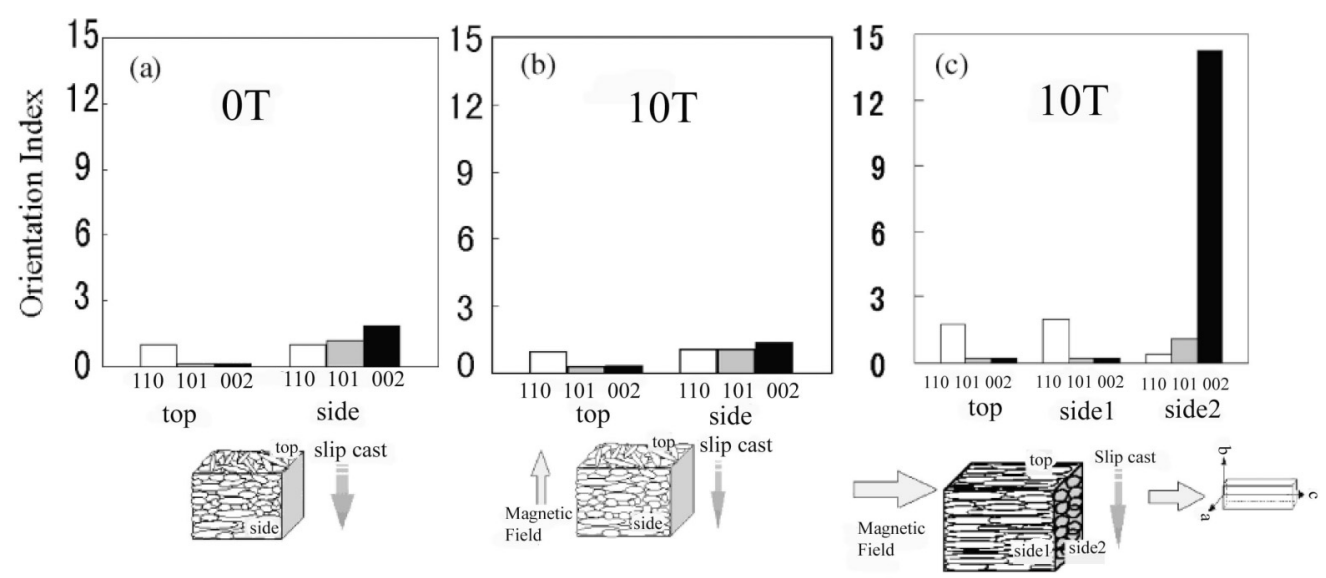

Fig. 3. Orientation indices of the peaks of (110), (101) and (002) and schematic of alignment of titania whisker. 
persed system when the crystal size is large enough.

In the case of Pa magnetic field as shown in Fig. 2(b), similar result to those without high magnetic field has been obtained. As shown in Table 2, under Pa magnetic field, all of $a, b$ and $c$ planes appear in each plane of a sample. In this case, we cannot get oriented structure by the magnetic field. These results are in accordance with the experimental results as shown in Figs. 2(b) and 3(b), where the orientation indices show no difference between the top plane and the side plane. And the indices (110), (101) and (002) show almost the same intensity, which indicates crystal alignment didn't occur by imposition of the high magnetic field and all of $a, b$ and $c$ planes appear on each plane of the sample. On the other hand, according to Table 2, under Pe magnetic field, $c$-axis can be uniaxially oriented in the side 2 plane while $a, b$ planes appear in the top and side 1 planes. These results are confirmed by the experiment results as shown in Figs. 2(c) and 3(c). The intensity of (110) is high while those of (101) and (002) are low in the top and side planes. And there is almost no difference between the top plane and the side 1 plane. That is, both of $a$ and $b$ planes appear on the top and side planes. While in the side 2 plane, which is perpendicular to the magnetic field, the intensity of (002) is very high. In other words, $c$ plane prefers to appear on the side 2 plane, which indicates the $c$-axis of the whisker is aligned parallel to the magnetic field direction.

\section{Conclusion}

In our research, the theoretical analysis indicates that the expected crystal alignment is different when the gravity force is taken into account. For a spherical crystal, the gravity force will not attribute to its rotation. However, for a large rod or plane shape crystal, the crystal orientation largely depends on the imposition manner of the high magnetic field. It is worth to point out that the theoretical derivation has well been confirmed by the experimental results, which is just a practical case appeared in the theoretical results. Thus, it can be concluded that the theoretical derivation is credible.

\section{Acknowledgment}

This research was supported by the 21 st COE program "The Creation of century Nature-Guided Materials Processing" and Grant-in-Aid for Scientific Research on Priority Areas (S), 13852013 of Ministry of Education, Culture, Sports, Science and Technology.

\section{REFERENCES}

1) H. Morikawa, K. Sassa and S. Asai: Mater. Trans. JIM, 39 (1998), 814.

2) K. Inoue, T. Marukawa, K. Sassa, Y. Yokogawa, Y. Sakka, M. Okido and S. Asai: Key Eng. Mater., 240-242 (2003), 513.

3) X. Y. Lu, A. Nagata, K. Watanabe, T. Nojima, K. Sugawara, S. Hanada and S. Kamada: Physica C, 392 (2003), No. 1, 453.

4) C. S. He, Y. D. Zhang, X. Zhao, L. Zuo, J. C. He, K. Watanabe, T. Zhang, G. Nishijima and C. Esling: Adv. Eng. Mater, 50 (2003), No. $8,579$.

5) W. P. Chen, H. Maeda, K. Watanabe and M. Motokawa: Physica C, 337 (2000), No. 1-4, 160.

6) J. M. Ferreira, M. B. Maple, H. Zhou, R. R. Hake, B. W. Lee, C. L. Seaman, M. V. Kuric and R. P. Guertin: Appl. Phys. A, 47 (1988), 105.

7) Y. Sakka, T. S. Suzuki, N. Tanabe, S. Asai and K. Kitazawa: Jpn. J. Appl. Phys., 41 (2002), L1416.

8) K. S. Willson and J. A. Rogers: Technical Proc. of Am. Electroplaters Soc., 51 (1964), 92.

9) C. Wu, S. Li, K. Sassa, Y. Chino, K. Hattori and S. Asai: Mater. Trans., 46 (2005), No. 6.

10) S. Li, K. Sassa, K. Iwai and S. Asai: Mater. Trans., 45 (2004), 3124.

11) T. S. Suzuki and Y. Sakka: Jpn. J. Appl. Phys., 41 (2002), L1272.

12) Y. Sakka and T. S. Suzuki: J. Ceram. Soc., Jpn., 113 (2005), 26. 